\title{
Quantum Brownian motion for periodic coupling to an Ohmic bath
}

\author{
J. Piilo, S. Maniscalco, and K.-A. Suominen \\ Department of Physics, University of Turku, FI-20014 Turun yliopisto, Finland
}

(Dated: May 15, 2019)

\begin{abstract}
We show theoretically how the periodic coupling between an engineered reservoir and a quantum Brownian particle leads to the formation of a dynamical steady state which is characterized by an effective temperature above the temperature of the environment. The average steady state energy of the system has a higher value than expected from the environmental properties. The system experiences repeatedly a non-Markovian behavior - as a consequence the corresponding effective decay for long evolution times is always on average stronger than the Markovian one. We also highlight the consequences of the scheme to the Zeno-anti-Zeno crossover which depends, in addition to the periodicity $\tau$, also on the total evolution time of the system.
\end{abstract}

PACS numbers: $03.65 \mathrm{Yz}, 03.65 \mathrm{Xp}$

\section{INTRODUCTION}

Fundamental research on open quantum systems has traditionally focused on interactions between the reduced system and its natural environment 1, 2]. Recent theoretical and experimental developments in engineering the properties of the environments open a new avenue by creating artificial controlled reservoirs with whom the reduced system interacts [3, 4, [5]. These developments make possible, e.g., to observe directly the appearance of decoherence [4, 5] and to study non-Markovian dynamics of open systems in new regimes [6, 7]. Moreover, reservoir engineering and environment mediated schemes allow the indirect control of quantum systems [8, 9] the quantum control being the essential ingredient in the development of quantum simulators. Generally speaking, the importance of the open system studies stems from their central role in understanding the quantumclassical border [10, 11, 12] and from the role of decoherence as an obstacle for creating quantum information processors [13, 14]. Reservoir engineering provides a so far largely unexplored way to approach these issues which have both fundamental and applicative character.

We focus on the reservoir engineering schemes for a paradigmatic open system model, i.e. quantum Brownian motion (QBM) 1, 2, 15, 16, 17, 18, 19], which has a wide variety of applications ranging from quantum optics [20] and nuclear physics [21] to chemistry [22]. An earlier study has revealed various types of non-Markovian dynamics of QBM for structured reservoirs [6], i.e. timeindependent engineered reservoirs [23]. Here, we concentrate on a scheme where the system is forced repeatedly to experience non-Markovian behavior due to the structured reservoir. We show that this corresponds to the case when the engineered reservoir is periodically switched off and on - and can also be seen as an initial step towards more sophisticated time-dependent reservoir engineering techniques. We call this switch off-on scheme as shuttered reservoir and consider the cases where the duration of each period is on the nonMarkovian time-scale [24].

So far time-dependent reservoir engineering schemes have been considered mostly for few-level systems 25]. Here, we give an example of the effects of periodic systemenvironment interaction for a more complicated quantum system, namely harmonic oscillator. Our results can find applications, e.g., in the field of linear quantum amplifiers. In that context, the switching off-on action corresponds to shuttering the pumping lasers, which may lead to the interesting effects such as the enhancement or the reduction of the gain of the amplified field [26].

It is worth noting that artificial engineered reservoirs has been recently created for trapped ion system by applying electric noise to the trap electrodes and by using laser light [4, 5]. Thus, one can think of creating shuttered reservoirs by shuttering the noise which is applied to trap electrodes or by shuttering the laser light.

The theoretical description of the system dynamics, that we consider, is based on the recursive use of the appropriate master equation and allows analytical solutions to be found. For a small number of shuttering periods, one can control the appearance of the quantum Zeno (QZE) and anti-Zeno effects (AZE) 28, 29, 30] for the reduced system by tailoring the properties of the environment [7]. In this paper we concentrate on the intermediate time dynamics (intermediate number of periods) and on the steady state properties of the reduced system for long time (large number of periods). We show that for a large number of cycles, the system always reaches a steady state which is not in thermal equilibrium with the environment at temperature $T$ but can be described by an effective temperature $T_{\text {eff }}>T$ and by the corresponding thermal state. The steady state value of the average system energy is given by the ratio of the effective time averaged diffusion and dissipation constants.

The scheme has also interesting fundamental consequences for the appearance of QZE and AZE. As was shown in Ref. 7], for a small number of cycles, one can control the appearance of the QZE or AZE by changing the environment parameters. In this paper we show that for large number of cycles the system always experiences an AZE. In other words, the decay of the system is stronger than the Markovian one for large number of periods. Thus, for short evolution times the system may 
experience QZE which for long times turns to AZE. This demonstrates that the appearance of quantum Zeno or anti-Zeno effects in QBM depends also on the total evolution time of the system (or number of measurements) and demonstrates the richness of Zeno dynamics in QBM when compared to more simple systems, e.g, two-level atoms.

The paper is organized in the following way. Section III introduces the quantum Brownian motion model for a harmonically bound particle and introduces the basic properties and parameters for the structured reservoirs we use. These form the framework for Sec. III in which the formal treatment of open system with shuttered reservoir is carried out leading to the concept of recursive master equation. The results for the heating function dynamics are presented in Sec. IV and the discussion concludes the paper in Sec. V.

\section{QUANTUM BROWNIAN MOTION}

We consider a harmonic oscillator linearly coupled with a reservoir modelled as an infinite set of non-interacting oscillators 1, 2, 15, 16, 17, 18, 19]. The dynamics of a damped harmonic oscillator is described, in the secular approximation, by means of the following generalized master equation in the interaction picture [6, 31]

$$
\begin{aligned}
& \frac{d \rho(t)}{d t}=\frac{\Delta(t)+\gamma(t)}{2}\left[2 a \rho(t) a^{\dagger}-a^{\dagger} a \rho(t)-\rho(t) a^{\dagger} a\right] \\
& +\frac{\Delta(t)-\gamma(t)}{2}\left[2 a^{\dagger} \rho(t) a-a a^{\dagger} \rho(t)-\rho(t) a a^{\dagger}\right] .
\end{aligned}
$$

In this equation, $a$ and $a^{\dagger}$ are the annihilation and creation operators, and $\rho(t)$ is the reduced density matrix of the system harmonic oscillator. The non-Markovianity is characterized by the time dependence of the coefficients $\Delta(t)$ and $\gamma(t)$ appearing in the master equation - these are known as diffusion and dissipation coefficients, respectively [6, 31].

The diffusion coefficient appearing in the master equation (1), to second order in the dimensionless coupling constant $g$, can be written in the units of $\hbar$ as $[\underline{6}, 16]$

$$
\begin{aligned}
\Delta(t)= & 2 g^{2} k_{\mathrm{B}} T \frac{r^{2}}{1+r^{2}}\left\{1-e^{-\omega_{c} t}\left[\cos \left(\omega_{0} t\right)\right.\right. \\
& \left.\left.-(1 / r) \sin \left(\omega_{0} t\right)\right]\right\},
\end{aligned}
$$

where the assumption of the high-temperature reservoir, $\bar{n}=k_{\mathrm{B}} T / \omega_{0} \gg 1$ has been used. The dissipation coefficient $\gamma(t)$ can be written as

$$
\gamma(t)=\frac{g^{2} \omega_{0} r^{2}}{r^{2}+1}\left\{1-e^{-\omega_{c} t}\left[\cos \left(\omega_{0} t\right)+r \sin \left(\omega_{0} t\right)\right]\right\} .
$$

Above, $r=\omega_{c} / \omega_{0}$ is the ratio between the environment cut-off frequency $\omega_{c}$ and the oscillator frequency $\omega_{0}, k_{\mathrm{B}}$ the Boltzmann constant, and $T$ the temperature of the environment.

A commonly used environment for open quantum systems is described by an Ohmic reservoir spectral density with the Lorentz-Drude cut-off [1]

$$
J(\omega)=\frac{2 \omega}{\pi} \frac{\omega_{c}^{2}}{\omega_{c}^{2}+\omega^{2}} .
$$

The spectral distribution is given by

$$
\begin{aligned}
I(\omega) & =J(\omega)\left[n_{e}(\omega)+1 / 2\right] \\
& =\frac{\omega}{\pi} \frac{\omega_{c}^{2}}{\omega_{c}^{2}+\omega^{2}} \operatorname{coth}(\omega / K T),
\end{aligned}
$$

where $n_{e}(\omega)$ is the occupation of the environment mode at frequency $\omega$ and Eq. (4) has been used. For high $T$, Eq. (5) becomes

$$
I(\omega)=\frac{2 k_{\mathrm{B}} T}{\pi} \frac{\omega_{c}^{2}}{\omega_{c}^{2}+\omega^{2}} .
$$

The central parameter $r=\omega_{c} / \omega_{0}$ describes how onresonant the oscillator is with the reservoir. When $r>$ 1 , intensive part of the environment spectrum overlaps with the oscillator frequency and the decay coefficients $\Delta(t) \pm \gamma(t)>0$ for all times. Consequently, the master equation is of Lindblad-type [32, 33]. When $r<1$, the most intense part of the environment spectrum lies in the small frequency range and the on-resonant intensity is small. The decay coefficients $\Delta(t) \pm \gamma(t)$ acquire temporarily negative values and the master equation is of non-Lindblad-type 27].

The full solution of the master equation (11) can be found e.g. in Refs. 6, 31]. In what follows we study the time evolution of the heating function $\langle n(t)\rangle$ with $n=a^{\dagger} a$ quantum number operator. The dynamics of $\langle n(t)\rangle$ depends only on the diffusion coefficient $\Delta(t)$ and on the classical damping coefficient $\gamma(t)$ [31]. Furthermore, the quantum number operator $n$ belongs to a class of observables not influenced by the secular approximation [18, 31].

The solution for the heating function, valid for all times and all initial states, is given by [6, 27]

$$
\langle n(t)\rangle=e^{-\Gamma(t)}\langle n(0)\rangle+\frac{1}{2}\left(e^{-\Gamma(t)}-1\right)+\Delta_{\Gamma}(t),
$$

where the quantities $\Delta_{\Gamma}(t)$ and $\Gamma(t)$ are defined in terms of the diffusion and dissipation coefficients $\Delta(t)$ and $\gamma(t)$ respectively as follows

$$
\begin{aligned}
\Gamma(t) & =2 \int_{0}^{t} \gamma\left(t_{1}\right) d t_{1}, \\
\Delta_{\Gamma}(t) & =e^{-\Gamma(t)} \int_{0}^{t} e^{\Gamma\left(t_{1}\right)} \Delta\left(t_{1}\right) d t_{1} .
\end{aligned}
$$

For short times the system experiences non-Markovian dynamics and for long times, after Markovian heating, the system reaches a thermal steady state with its environment. This typical dynamics is modified in the context of the scheme presented in the next section. 


\section{SHUTTERED RESERVOIR AND RECURSIVE MASTER EQUATION}

We are interested in the reduced system dynamics when its engineered artificial reservoir is switched off and on in a repeated manner with period $\tau$ - for this purpose we use the term shuttered reservoir. At the end of each period the engineered reservoir is switched off, and switching on initiates a new time evolution period for the system. Consequently, each switching off and on process will reset the correlations between the system and the environment. For an experimental realization of the scheme, one can, e.g., uncouple the system from the engineered bath in a periodic way. The duration of the free evolution period has to be chosen long enough to avoid correlations between different system-bath coupling periods, i.e., the duration of the free evolution has to exceed the bath correlation time.

By resetting the correlations between the system and the environment, the system oscillator is forced to experience non-Markovian dynamics in a periodic way. Consequently, the system remains repeatedly in the nonMarkovian regime. Following this scheme, the solution for the reduced density matrix and the heating function dynamics can be obtained by the recursive use of the master equation given by Eq. (11). In other words, the master equation is solved for each evolution period by using as an initial state of the system the one obtained at the end of the previous period. In the case we consider, the free evolution between system-reservoir interaction periods can be neglected in the calculations [34].

This recursive use of the master equation (1) leads, assuming an initial Fock-state, to the following equation for the density matrix of the reduced system:

$$
\begin{aligned}
\frac{d \rho(t)}{d t} & =\gamma_{1}(\tau)\left[a \rho(t) a^{\dagger}-\frac{1}{2} a^{\dagger} a \rho(t)-\frac{1}{2} \rho(t) a^{\dagger} a\right] \\
& +\gamma_{-1}(\tau)\left[a^{\dagger} \rho(t) a-\frac{1}{2} a a^{\dagger} \rho(t)-\frac{1}{2} \rho(t) a a^{\dagger}\right] .
\end{aligned}
$$

Here, time $t=m \tau$ where $m$ indicates the number of the period. The decay coefficients are written as

$$
\gamma_{1}(\tau)=\frac{1}{\tau} \int_{0}^{\tau} d t^{\prime}\left[\Delta\left(t^{\prime}\right)+\gamma\left(t^{\prime}\right)\right]
$$

and

$$
\gamma_{-1}(\tau)=\frac{1}{\tau} \int_{0}^{\tau} d t^{\prime}\left[\Delta\left(t^{\prime}\right)-\gamma\left(t^{\prime}\right)\right]
$$

It can be shown that the master equation (10) corresponds formally to the case where the reduced system dynamics is modified by periodic non-selective measurements. Thus, our shuttered reservoir and initial state are equivalent to performing periodically non-selective measurements of the energy of the oscillator [35]. For a formal study of non-selective measurements and master equations, see Ref. [36].

We are mostly interested in the case where the oscillator is initially in its ground Fock-state $|n=0\rangle$ (such as laser cooled single trapped ion) though the results for the steady state presented in the following are valid for all initial states. For an initial ground state, the solution of the master equation (10), in terms of the quantum characteristic function $(\mathrm{QCF}) \chi$ reads

$$
\chi_{\mathrm{t}}(\xi)=\exp \left\{-\left[\langle n(t)\rangle+\frac{1}{2}\right]|\xi|^{2}\right\},
$$

where the heating function is given by

$$
\langle n(t=m \tau)\rangle=\left(\frac{\Delta_{\Gamma}(\tau)}{1-e^{-\Gamma(\tau)}}-\frac{1}{2}\right)\left(1-e^{-m \Gamma(\tau)}\right) .
$$

The solution given in Eq. (13) is obtained by using certain algebraic properties of the superoperators of the corresponding generalized master equation, for more details see Refs. 66, 27, 31]. Moreover, the QCF of Eq. (13) corresponds to a thermal state at all times (c.f. Ref. [37]) and its time-dependence is given by $\langle n(t)\rangle$.

Eq. (14) reveals that there exists a steady state value for the heating function, $\langle n\rangle_{s}$, and its value can be easily obtained by taking the limit of number $m$ of periods going to infinity. We obtain from Eq. (14)

$$
\langle n\rangle_{s}=\lim _{m \rightarrow \infty}\langle n(t)\rangle=\frac{\Delta_{\Gamma}(\tau)}{1-e^{-\Gamma(\tau)}}-\frac{1}{2} .
$$

It is important to notice that the steady state value depends on the duration $\tau$ of the periods. Moreover, since $\Delta$ and $\gamma$ depend on $r$, consequently also $\Gamma$ and $\Delta_{\Gamma}$ depend on $r$ [c.f. Eqs. (8) and (9)], and the steady state value may also depend on the form of the environmental spectral density applied in each period, and not only on its temperature $T$.

As a cross check, it is easy to see that with increasing duration of the period $\tau$, we obtain correctly the result without shuttering. The denominator in Eq. (15), $1 /\left(1-e^{-\Gamma(\tau)}\right)$, goes to unity with increasing $\tau$ and we obtain $\langle n\rangle_{s}=\Delta_{\Gamma}(\tau)-1 / 2$. This matches with the long time result without the shuttering which can be calculated by taking the limit $t \rightarrow \infty$ in Eq. (7).

To gain more physical insight, we expand $\exp [-\Gamma(\tau)]$ which appears explicitly and implicitly in Eq. (15), with respect to $\Gamma$. Keeping the terms to lowest order in $\Gamma$, and neglecting the term $1 / 2$ since we consider high temperature case, we obtain

$$
\langle n\rangle_{s}=\frac{1}{2} \frac{\int_{0}^{\tau} d t^{\prime} \Delta\left(t^{\prime}\right)}{\int_{0}^{\tau} d t^{\prime} \gamma\left(t^{\prime}\right)} .
$$

Moreover, this equation is valid also in the Markovian limit since with the Markovian values for $\Delta$ and $\gamma$ we obtain $\langle n\rangle_{s}=k_{\mathrm{B}} T / \omega_{0}$, which matches the high temperature Markovian result [38]. 
Equation (16) demonstrates that the ratio between the time averaged diffusion and dissipation gives the steady state value of the average system energy in the presence of shuttered reservoirs. Here, the average is taken over a single period of duration $\tau$. The system is not in thermal equilibrium with its environment and has an effective temperature $T_{\text {eff }}$ which is different than the temperature $T$ of the environment. Moreover, the steady state average energy of the system can be controlled by the period duration $\tau$ and the environment "on-resonance" parameter $r$.

The steady state of the system is a thermal state, see Eq. (13), with $\langle n(t)\rangle=\langle n\rangle_{s}$. The corresponding effective temperature can be written as

$$
T_{\text {eff }}=\frac{\omega_{0}}{k_{\mathrm{B}}}\langle n\rangle_{s} .
$$

This differs from the environment temperature $T$ since $\langle n\rangle_{s} \neq \bar{n}$. Moreover, since the system reaches an effective thermal state, it eventually fulfills the detailed balance condition with appropriate modifications to the transition rates given by the shuttered reservoir.

We have presented above the formalism and the crosschecks for its validity. In the following section we continue with the results for the heating function dynamics from Eq. (14) and the steady state values of the average system energy from Eq. (15).

\section{THE SYSTEM DYNAMICS}

\section{A. Short and intermediate time dynamics}

Figure 1 displays the heating function dynamics over a couple of first shuttering periods. It illustrates how the system exhibits (a) Zeno and (b) anti-Zeno effect. For various types of non-Markovian dynamics with traditional unshuttered reservoirs in this regime see Fig. 1 in Ref. [6] and for a study of the Zeno - anti-Zeno crossover see Ref. [7]. The key aspect for this paper is that both effects are possible for short evolution times. Moreover, one can control which one appears by controlling the periodicity $\tau$ and the properties of the environment. Note that the figure includes also the non-Markovian dynamics within each period and not only the coarse graining in $\tau$ [c.f. Eq. (14)].

Examples of the heating function dynamics for intermediate times, when the system approaches the steady state, are displayed in Fig. 2. The results demonstrate clearly that dynamics which begins with reduced heating, and therefore as Zeno-type for short evolution times, becomes anti-Zeno for long times. This can be observed as a crossing between the dotted and solid lines in Fig. 22(a) which shows in detail the passage from Zeno to anti-Zeno region.

Moreover, Fig. 2(b) demonstrates that the approached steady state value for the dotted line is around 3.5 times
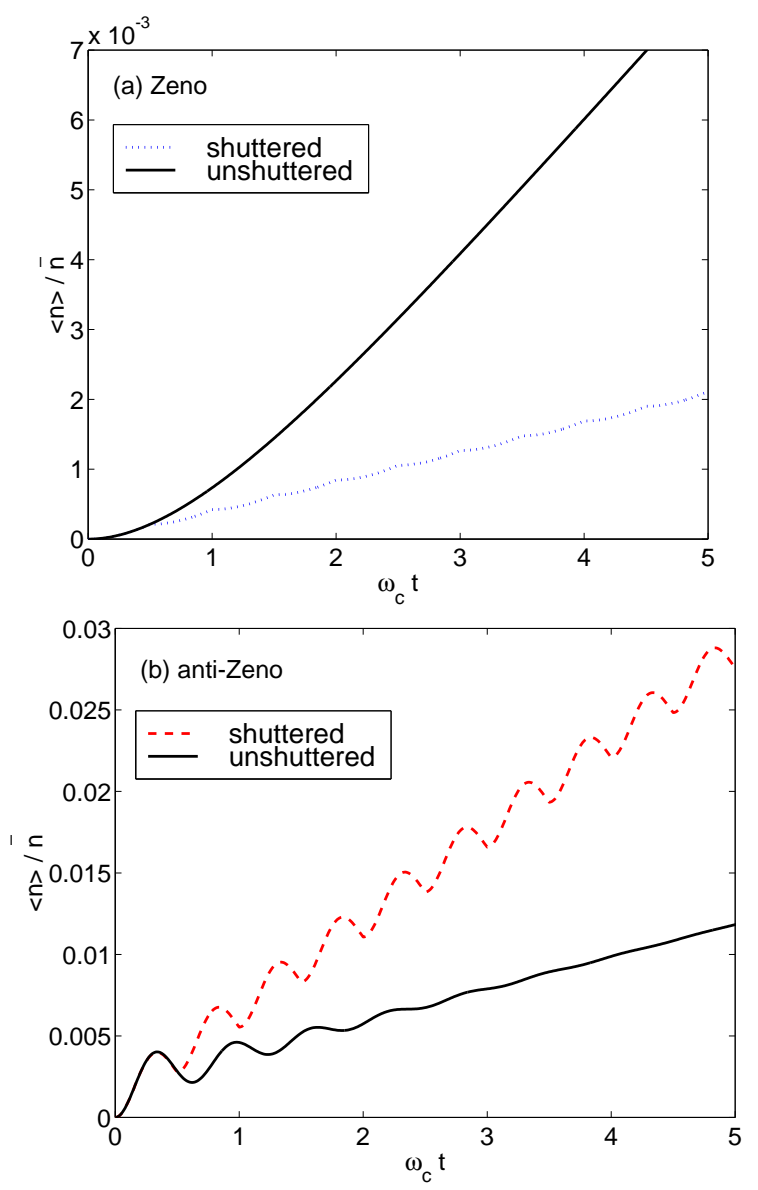

FIG. 1: (Color online) The short time behavior of the heating function displaying (a) Zeno and (b) anti-Zeno effect. In the Zeno effect, the shuttered reservoir reduces the heating of the oscillator while for the anti-Zeno case the heating is enhanced when compared to the dynamics with the traditional unshuttered reservoir. The parameters are $g=0.1, \tau=0.5 / \omega_{c}$, $\bar{n}=k_{\mathrm{B}} T / \omega_{0}=10$. In (a) $r=10$ and (b) $r=0.1$.

the one expected from the temperature of the environment. Furthermore, it is striking that there exists a crossing between the dotted and dashed lines in Fig. 2 (b). In another words, the dynamics which starts as Zeno-type, shows for long times more pronounced anti-Zeno character than the case which already initially begins as antiZeno type. As a consequence, the highest steady state values of the energy for long times are reached for the parameters that in short times give QZE and reduced decay.

The results above clearly demonstrate that the appearance of QZE and AZE is time-dependent in QBM. There exists a crossover between the two effects in time. The fact that the appearance of these effects depends on the total evolution time of the system, in addition of the periodicity $\tau$, is in contrast to more simple systems like two-level atoms. It has been shown in Ref. [7], which studied the controlling of the Zeno and anti-Zeno effects in QBM for short times, that the appearance of 

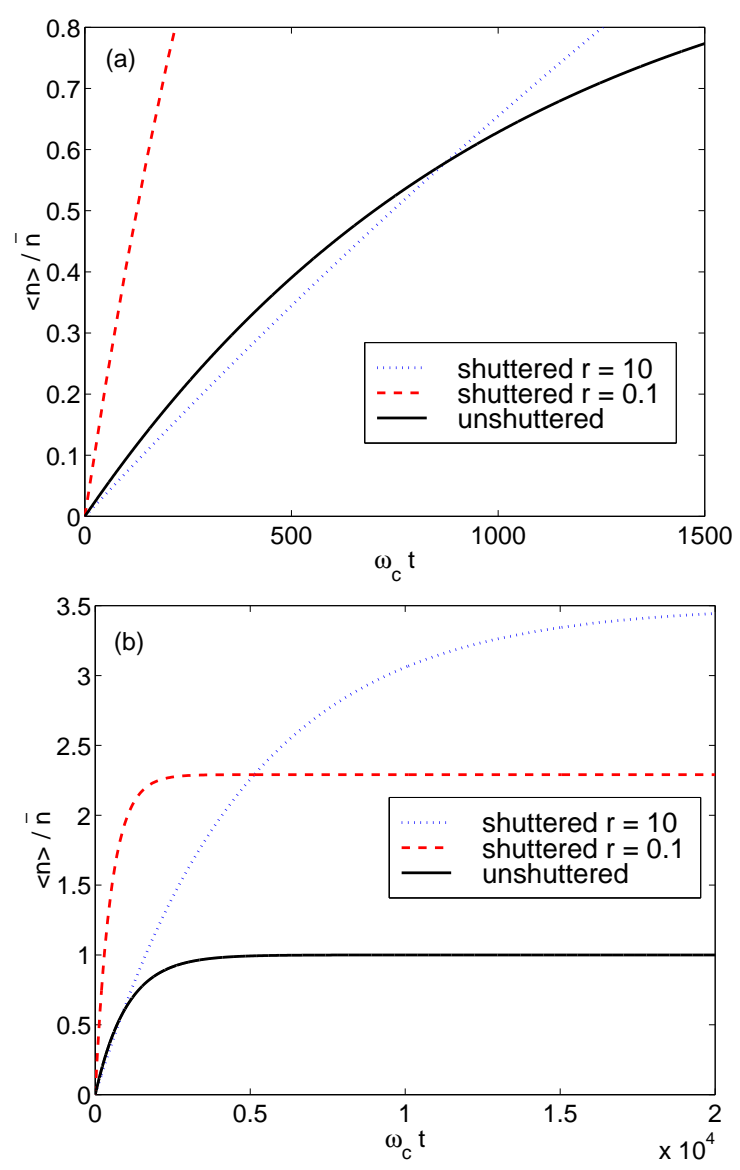

FIG. 2: (Color online) The heating function dynamics for intermediate times when the system appproaches a steady state. In panel (a) $0 \leqslant \omega_{c} t \leqslant 0.15 \times 10^{4}$ which shows the small time blow up of panel (b) where $0 \leqslant \omega_{c} t \leqslant 10^{4}$. The parameters correspond to those used in Fig. 1 1 except $\tau=1 / \omega_{c}$. The solid line is for an unshuttered reservoir showing the thermalization to $\langle n\rangle / \bar{n}=1$ in panel (b). The dashed line is for the case of pure anti-Zeno dynamics by shuttered reservoir for all times. The dotted line is for shuttered reservoir when the dynamics begins as Zeno-type (reduced heating) but crosses later to anti-Zeno behavior (increased heating). This crossing between the solid and dotted lines, which occurs at $\omega_{c} t \simeq 800$, is shown in detail in panel (a).

the QZE and AZE depends on the quantum number $n$ for QBM. Even though the time averages of the effective decay coefficients remain fixed, this $n$-dependence allows the crossover from Zeno to anti-Zeno effect to occur with time. When the system heats, the expectation value $\langle n\rangle$ increases with time, and the system experiences a crossover from Zeno to anti-Zeno region. This is not possible for a two-level system and demonstrates that the Zeno-anti-Zeno phenomena has more variety in QBM than in more simple systems.

\section{B. Long-time dynamics and steady state}

The results presented in the previous subsection suggest that the heating function with shuttered reservoirs approaches asymptotically values which are higher than with the unshuttered reservoirs. Actually, this is also indicated by the analytic solution given in Eq. (14) where the denominator is always between 0 and 1 . The system which is forced repeatedly to go through the nonMarkovian behavior, experiences enhanced dissipation compared to the Markovian one. The system reaches a steady state since the time dependent diffusion and dissipation coefficients, $\Delta$ and $\gamma$ respectively, have well defined time averaged values over $\tau$.

By using Eq. (15) one can calculate the steady state system energy as a function of $\tau, r$, and the coupling constant $g$. The magnitude of the coupling constant, in the region of the validity of the model (weak coupling $g \ll 1$ ) does not affect the steady state energy. Moreover, $\langle n\rangle_{s}$, as a function of $r$, varies in a rather small range for $r \lesssim 1$ and then reaches a stable value for increasing $r$. Thus, the most interesting parameter here is the shuttering interval $\tau$. This conclusion can be reached also by making a series expansion of $\langle n\rangle_{s}$, with respect to small $\tau$. The dominant term contains only $\tau$, and the parameters $r$ and $g$ come into play only in the higher order terms.

Figure 3 displays the steady state $\langle n\rangle_{s}$ as a function of $\tau$, where for other parameters some convenient values has been chosen. As expected, for increasing $\tau$, the average energy of the steady state approaches the one with unshuttered reservoir and corresponds to the temperature of the environment. When the duration of the periods $\tau$ is decreased, the steady state energy of the system increases rapidly. This can be explained by studying the behavior of $\Delta$ and $\gamma$ as a function of time. For the typical spectral density we use in this paper [c.f. Eq. (44)], $\Delta$ initially increases in the non-Markovian region faster than $\gamma$ [c.f. Eqs. (2) and (3)]. The feature is enhanced in the time averaged values of these quantities when $\tau$ decreases and demonstrates quantitatively the behavior seen in Fig. 3,

Naturally, the energy conservation has to be satisfied. It requires work to periodically shutter the reservoir. In other words, it takes work to periodically couple the system with the reservoir. This can be seen as the origin of the increased system energy and the energy is conserved since the minimum work required exceeds the oscillator energy [39].

\section{DISCUSSION AND CONCLUSIONS}

The creation of artificial environments and the reservoir engineering techniques open new avenues to study the non-Markovian dynamics of open quantum systems and to improve the capability for the control of quantum systems. We have taken here an initial step towards time-dependent reservoir engineering by considering the 


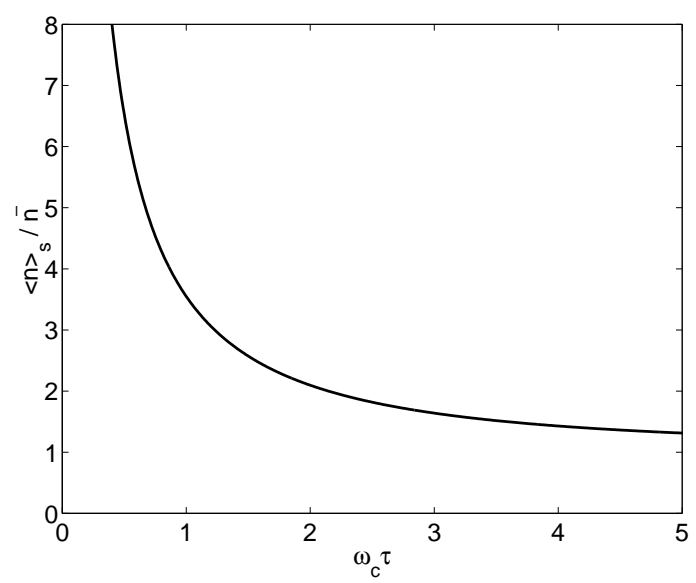

FIG. 3: The steady state value of the heating function $\langle n\rangle_{s}$ as a function of the shuttering period $\tau$. Parameters are $\alpha=0.1$, $r=10, \bar{n}=10$.

case when a structured reservoir is switched on and off in a periodic manner (shuttered reservoir).

The results for quantum Brownian motion show that the interaction of the reduced system with the shuttered reservoir changes the system dynamics drastically compared to the conventional dynamics. For short times the system may exhibit Zeno or anti-Zeno behavior depending on the properties of the environment. A striking dynamical feature arises for long-times - the system always reaches a steady state in which the average system energy is larger than the one corresponding to the the temperature of the environment. The shuttered reservoir forces the system to experience the non-Markovian behavior periodically and the steady state properties are consequently given by the time averaged properties of the diffusion and dissipation between successive reservoir shuttering events.

One of the interesting consequences is that the appearance of Zeno or anti-Zeno effects becomes dependent on the total evolution time of the system. What starts as a Zeno effect for short and intermediate times turns to antiZeno effect when the steady state regime is approached. The Zeno studies in simple systems, such as a two-level system, show how either Zeno or anti-Zeno effect appears. Here, we have demonstrated that a crossing between the two effects occurs with time and in QBM the Zeno phenomena is very rich.

In the future, advanced reservoir engineering techniques may allow new ways to implement quantum control. We have shown here that shuttered reservoir gives rise to interesting dynamical effects. A next step is to study the case where the properties of the environment remain the same for a given "on-period" but vary between different periods. Very precise ways to control the system properties by using reservoir engineering combined with laser cooling may open new possibilities for the development of general quantum simulators for open systems whose dynamics is traditionally tedious to solve by analytical means.

\section{Acknowledgments}

This work has been supported by the Academy of Finland (projects 108699, 115682, 115982) and the Magnus Ehrnrooth Foundation.
[1] U. Weiss, Quantum Dissipative Systems (World Scientific Publishing, Singapore, 1999).

[2] H.-P. Breuer and F. Petruccione, The Theory of Open Quantum Systems (Oxford University Press, Oxford, 2002).

[3] J. F. Poyatos, J. I. Cirac, and P. Zoller, Phys. Rev. Lett. 77, 4728 (1996).

[4] C.J. Myatt, B.E. King, Q.A. Turchette, C.A. Sackett, D. Kielpinski, W.M. Itano, C. Monroe, and D.J. Wineland, Nature (London) 403, 269 (2000).

[5] Q.A. Turchette, C. J. Myatt, B. E. King, C. A. Sackett, D. Kielpinski, W. M. Itano, C. Monroe, and D. J. Wineland, Phys. Rev. A 62, 053807 (2000).

[6] S. Maniscalco, J. Piilo, F. Intravaia, F. Petruccione, and A. Messina, Phys. Rev. A 70, 032113 (2004).

[7] S. Maniscalco, J. Piilo, and K.-A. Suominen, Phys. Rev. Lett. 97, 130402 (2006).

[8] P. Rabl, A. Shnirman, and P. Zoller, Phys. Rev. B 70, 205304 (2004).

[9] R. Romano and D. D'Alessandro, Phys. Rev. Lett. 97, 080402 (2006).

[10] W. H. Zurek, Los Alamos Science 27, 86 (2002).
[11] W. H. Zurek, Rev. Mod. Phys. 75, 715 (2003).

[12] E. Joos, H.-D. Zeh, C. Kiefer, D. Giulini, J. Kupsch, and I.-O. Stamatescu, Decoherence and the Appearence of a Classical World in Quantum Theory, 2nd ed. (SpringerVerlag, Berlin, 2003).

[13] M. A. Nielsen and I. L. Chuang, Quantum Computation and Quantum Information (Cambridge University Press, Cambridge, 2000).

[14] S. Stenholm and K.-A. Suominen, Quantum Approach to Informatics (Wiley, Hoboken, New Jersey, 2005).

[15] R. P. Feynman and F. L. Vernon, Ann. Phys. (N.Y.) 24, 118 (1963).

[16] A. O. Caldeira and A. J. Leggett, Physica A 121, 587 (1983).

[17] F. Haake and R. Reibold, Phys. Rev. A 32, 2462 (1985).

[18] H. Grabert, P. Schramm, and G.-L. Ingold, Phys. Rep. 168, 115 (1988).

[19] B. L. Hu, J. P. Paz, and Y. Zhang, Phys. Rev. D 45, 2843 (1992).

[20] L. Mandel and E. Wolf, Optical Coherence and Quantum Optics (Cambridge University Press, Cambridge, 1995).

[21] I. Joichi, Sh. Matsumoto, and M. Yoshimura, Phys. Rev. 
A 57, 798 (1998).

[22] P. Hänggi, P. Talkner, and M. Borkovec, Rev. Mod. Phys. 62, 251 (1990).

[23] By a time independent reservoir we mean the ones whose spectral properties remain unchanged with time.

[24] We have shown earlier that the non-Markovian time scale can be prolonged to an experimentally accessible regime [27].

[25] A. G. Kofman and G. Kurizki, Phys. Rev. Lett. 93, 130406 (2004).

[26] S. Maniscalco, J. Piilo, N. Vitanov, and S. Stenholm, Eur. Phys. J. D 36, 329 (2005).

[27] S. Maniscalco, J. Piilo, F. Intravaia, F. Petruccione, and A. Messina, Phys. Rev. A 69, 052101 (2004).

[28] B. Misra and E. C. G. Sudarshan, J. Math. Phys. (N.Y.) 18, 756 (1977).

[29] A. M. Lane, Phys. Lett. A 99, 359 (1983); W. C. Schieve, L. P. Horwits, and J. Levitan, Phys. Lett. A 136, 264 (1989); A. G. Kofman and G. Kurizki, Nature (London) 405, 546 (2000).

[30] P. Facchi, H. Nakazato, and S. Pascazio, Phys. Rev. Lett. 86, 2699 (2001); P. Facchi and S. Pascazio, in Progress in Optics, edited by E. Wolf (Elsevier, Amsterdam, 2001), Vol. 42, Chap. 3, p. 147.

[31] F. Intravaia, S. Maniscalco, and A. Messina, Phys. Rev. A 67, 042108 (2003).

[32] G. Lindblad, Commun. Math. Phys. 48, 119 (1976).
[33] V. Gorini, A. Kossakowski, and E. C. G. Sudarshan, J. Math. Phys. 17, 821 (1976).

[34] In our case, the state of the system is a thermal state at all times. Since a thermal state, being diagonal in the Fock-state basis, is not affected by free-evolution, the periods between two successive system-reservoir interaction periods can be neglected.

[35] Different measurement schemes, general initial states, their influence to the reduced system dynamics, and connection to dynamical decoupling formalism will be dealt with in a separate publication in preparation. In this paper, we demonstrate the inevitability of the "high energy" steady state and fundamental consequences for the Zeno - anti-Zeno crossover.

[36] P. Facchi, S. Tasaki, S. Pascazio, H. Nakazato, A. Tokuse, and D. A. Lidar, Phys. Rev. A 71, 022302 (2005).

[37] S. M. Barnett and P. M. Radmore, Methods in Theoretical Quantum Optics (Oxford University Press, Oxford, 1997).

[38] It is also possible to derive Eq. (16) by considering the decay rates between the Fock-states and assuming that in the steady state the average system energy does not change in time.

[39] G. W. Ford and R. F. O'Connell, Phys. Rev. Lett. 96, 020402 (2006). 